\title{
LA SEMIÓTICA EN CHILE
}

\author{
Rafael del Villar Muñoz
}

Universidad de Chile

There are three historical periods in the chilean semiotics. The first one corresponds to its birth (1969) associated with the ideological fight of society as a whole. The socond one (1975-1982) corresponds to the hegemony of an epistemological field whose fundamental demands are the gaps in specific theoretical fields: the literature and the visual aesthetics. The third period (1981 to this day), that incorporates a new epistemological region: the communications develops a rupture with the previous field, the questions are no longer originated in the theory but in the productive structure of society.

\section{SEMIÓTICA, RUPTURA EPISTEMOLÓGICA Y CAMPO TEÓRICO: SU ORIGEN EN CHILE DE 1969}

El nacimiento contemporáneo de la semiótica en los países desarrollados está directamente ligado a llenar una necesidad. Así, ella nace 
(o renace) en relación con una carencia, siendo los desequilibrios de funcionamiento de los dispositivos de acumulación del saber en campos teóricos concretos los que le dan un lugar. La semiótica no será un nuevo mirar sobre los fenómenos, ella planteará un no-visto por los otros, ella mirará lo que las otras disciplinas no han visto: el sentido, la significación que únicamente es posible de aprehenderse en el interior de un texto como totalidad. En consecuencia, la semiótica nace como una ruptura epistémica en relación a lo que la sociedad blanca es capaz de ver, en un momento histórico concreto. La teoría de la literatura había hecho, en relación al sentido, lecturas transversales de los textos poéticos: cada analista insertaba los datos en el interior de categorías ya establecidas; el problema que se planteaba era que las categorías eran diferentes según cada analista, por lo que el objeto real estaba fetichizado. Lo mismo ocurría en la teoría de la pintura, del teatro y de la arquitectura. La publicidad estaba dividida: el A.I.D.A. y el Análisis Motivacional, pero ninguna de las dos daban cuenta del mensaje; la publicidad producía conocimientos acerca del receptor (la percepción, las motivaciones), sin ligar los significantes del texto a dichos mundos posibles; había, entonces, un desequilibrio de funcionamiento entre una Estrategia Creativa Publicitaria construida a través de contenidos establecidos por el diagnóstico motivacional y la implementación concreta de piezas gráficas y spots, a los que no se les atribuía la calidad de vehiculizadores de la información. La teoría del cine estaba, también, en crisis: reflexiones a partir de la práctica misma, una filosofía de la acción que no constituía su propio objeto. En la música había una situación similar: heterogeneidad, incoherencia conceptual, una filosofía del hacer a partir del hacer. El psicoanálisis carecía de una metodología para aprehender las manifestaciones del inconsciente. La imposibilidad de aprehender el sentido correspondía a la lingüística, la sociología y la antropología: no podía más que producirse conocimientos a través del análisis de contenido, es decir según una perspectiva únicamente descriptiva.

Frente a esto, se pueden ver los temas de la semiótica de la primera generación: las condiciones necesarias de la manifestación del sentido (Greimas, Coquet, Courtés) y de su rechazo (Kristeva); la estructura, lectura y producción de los textos culturales (Eco, Barthes); la sintaxis y retórica visual de la publicidad (Péninou, Durand); el lenguaje y la gramática- retórica del cine (Metz), de la pintura (Marin), de la música (Ruwet, Nattiez); el lenguaje del inconsciente (Lacan, Laplanche, Lemaire); la estructura inconsciente que habla a través de los mitos (Lévi- Strauss), etc. 
Hay, entonces, una correspondencia entre los problemas que interesaron a la semiótica de la primera generación y los desequilibrios de funcionamiento de los dispositivos teóricos de una región epistémica concreta: la imposibilidad de las ciencias humanas y sociales de aprehender el sentido, porque éste sólo es posible establecerlo a partir de un texto, aunque él mismo sea por definición intertextual.

El nacimiento de la semiótica en Chile (fechado históricamente a fines de 1969) es totalmente diferente. Ella no llena una necesidad de los dispositivos teóricos de una región epistémica del saber, o los llena muy débilmente. Ella llena, sobre todo, las necesidades de ensoñación de la totalidad social, de la lucha ideológica. Habrá dos regiones epistemológicas: una región por nacer, la de la estética; y otra hegemónica, la de la crítica cultural.

En Chile, a fines de 1969 y hasta 1973, habrá una intensa actividad política. La sociedad será un campo de fuerza de las ideologías en lucha y la política será el centro de referencia de la sociedad global. En ese contexto nace la semiótica en Chile, insertándose en la crítica cultural en una región epistémica hegemónica: la semiótica llenará las necesidades de ensoñación de la sociedad global, siendo la lucha ideológica quien le dará un lugar.

Habrá dos centros de investigación que harán de la semiótica su nudo central. Habrá investigaciones, enseñantes, publicaciones y un lugar institucional:

-Área de Comunicaciones, Centro de Estudios de la Realidad Nacional (CEREN), Pontificia Universidad Católica de Chile, 1968-1973. Investigadores: Armand Mattelart, Michèle Mattelart, Mabel Piccini. Publicaciones: A. Mattelart: «Prefiguración de la ideología burguesa» (A. Mattelart, 1969); «El marco del análisis ideológico» (A. Mattelart, 1970a); «Estructura del poder informativo y dependencia» (A. Mattelart, 1970b); «La mitología de la juventud en un diario liberal»,(A. Mattelart, 1970c); « ¿Hacia una cultura de la movilización cotidiana? (A. Mattelart, 1971). Michèle Mattelart: «El nivel mítico de la prensa seudo-amorosa» (M. Mattalart, 1970a); «El conformismo revoltoso de la canción popular» (M. Mattelart, 1970b). Mabel Piccini: «El cerco de las revistas de ídolos» (Piccini, 1970).

-Departamento de Comunicaciones. Escuela de Artes de la Comunicación (EAC), Pontificia Universidad Católica de Chile, 19701975. Investigadores: Luis Felipe Ribeiro, Gisélle Munizaga, Consuelo Morel, Rina Alcalay, Rafael del Villar, Valerio Fuenzalida. Publicaciones: G. Munizaga: «Algunas ideas sobre lo ideológico en el cine» (Munizaga, 1972a); «¿Cuántas patas tiene un gato?» (Munizaga, 1972b); «La teleserie policial: una moral de la violencia» (Munizaga, 1975). C. Morel, F. Ossandon, V. Fuenzalida: «Más allá de la entretención de las teleseries. Búsqueda de la ideología en Bonanza y F.B.I. en Acción» (Morel, 
Ossandon y Fuenzalida, 1972). L.F. Ribeiro: «Apuntes sobre el problema lingüístico en la alfabetización» (Ribeiro, 1970); «Sobre la semantización de la sexualidad» (Ribeiro, 1972); «La estructura mítica de los discursos sobre la legalidad» (Ribeiro, 1972).

Una mirada sólo a los títulos precedentes nos permite detectar la profunda ligazón de la semiótica chilena, en sus inicios, con la crítica cultural, insertándose en una región epistémica hegemónica que constituirá el marco simbólico de referencia de la sociedad global.

Si analizamos la teoría semiótica puesta en acto en las investigaciones citadas, nos encontramos con la utilización de la semiótica greimasiana de la «Semántica Estructural» (Greimas, 1966): el modelo actancial, los recorridos figurativos, y el cuadrado semiótico; pero, sin una articulación metodológica, se trata de recorridos analíticos semióticos, más que de análisis semióticos propiamente tales.

Los presupuestos teóricos son los de la semiótica de la primera generación: se cree que aprehender la isotopía que habla en el texto, significa aprehender el universo de la interpretación al mismo tiempo que el universo inconsciente del sujeto generador. Es decir, se conceptualiza un lector pasivo: aprehender lo que los medios masivos nos hablan significa aprehender cómo la gente es manipulada por estos medios. De allí, el lugar ideológico hegemónico de esta primera semiótica chilena. La crítica encuentra en la semiótica una herramienta para aprehender el inconsciente hablado y el inconsciente leído, porque la semiótica misma no ha comprendido aún los procedimientos de lectura de los textos culturales. Es por ello que los temas de investigación son objetos críticos, en relación directa con la coyuntura política chilena entre 1969 y 1973.

Habrá otra región epistémica no hegemónica que llenará las necesidades de los dispositivos teóricos de la acumulación del saber en arquitectura, literatura y pintura; pero se trata de un trabajo de investigadores individuales, en el interior de su propio dominio, salvo el caso de la literatura, donde habrá un grupo de investigadores, dirigido por Nelson Osorio, José Varela (Universidad de Chile, sede Valparaíso) y René Jara (Universidad Católica de Valparaiso), quienes harán la edición (1972 a 1973) de la Revista Problemas de Literatura, con un fuerte contenido semiótico. Pero la regla será el estatus individual del proceso de construcción del conocimiento. Así, hay que citar las investigaciones semióticas de Luis Waisman sobre la arquitectura, en la Universidad de Chile, que darán origen al primer libro de semió- 
tica publicado en nuestro país: Semiología Arquitectónica (Waisman, 1974); las investigaciones de Iván Carrasco, en la Universidad Católica de Temuco (la teoría literaria le llevó a la semiótica, vía el estructuralismo, Revista Estilo, Temuco); las de Matus y Rabanales (semiolingüística, 1970); las de Manuel Jofré (semiótica de la literatura, 1972-1974) y las de Brugnoli y Ravanales (semiótica de la pintura), en la Universidad de Chile; tratándose siempre de investigadores individuales sin la institucionalización de un espacio semiótico social.

Otra característica de este tipo de investigaciones fue la de llenar las necesidades propias del desequilibrio de funcionamiento de la teoría literaria, de la pintura y de la arquitectura; es decir, una referencia a una región específica de la estética sin inserción en las necesidades de la sociedad global y sin conciencia clara, para cada dominio en particular, de las carencias de su propio dispositivo de acumulación del saber, sin existir conciencia de la necesidad semiótica.

En las mismas coordenadas históricas habrá otro Centro de Investigaciones Semióticas en el Instituto de Ciencias Sociales y Desarrollo (ICSD), de la Universidad Católica de Valparaíso (19701973), el que se ubicará a medio camino entre satisfacer las necesidades de la crítica ideológica y las necesidades propias de los dispositivos teóricos de la ciencia de la comunicación.

Investigadores: María Inés Silva, Eduardo Contreras, Rafael del Villar, Adriana Doñas. Publicaciones: E. Contreras, R. del Villar: «Comunicación e Ideología: Objeto, Teoría y Metodología» (Del VillarContreras, 1971). R. del Villar: Mensaje (forma y contenido) e ideología (Del Villar, 1971a); La concepción estructuralista de los mensajes (Del Villar, 1971b); La concepción estructuralista marxista de los mensajes (Del Villar, 1971c); «De cómo tanto la forma visual como su contenido son expresión de ideología» (Del Villar, 1972)». M.I. Silva: «Semiología del Western» (Silva, 1971).

En Chile, el Golpe Militar de 1973 significará un cambio sociopolítico en todo el contexto cultural nacional en el que el dispositivo semiótico hegemónico tenía su referente. Esto implicará la desaparición progresiva de los centros: CEREN y ICSD, en 1973; EAC, en 1975; y con ello, la desaparición del espacio de la crítica. Quedarán los investigadores individuales de la región epistemológica no hegemónica, que encontrarán un lugar, un espacio institucional, en el interior de la estética visual y de la literatura, en las universidades chilenas. 


\section{LA SEGUNDA SEMIÓTICA: RENACIMIENTO Y CAMPO TEÓRICO, 1975/ 1982}

Así, en el período 1975-1982, nos encontramos con una semiótica ya constituida en los dominios de la estética visual y la literatura. Los principios de su forma de funcionamiento serán similares a la semiótica de la primera generación en los países desarrollados, que llenará las necesidades producidas por los desequilibrios del aparato acumulador de conocimientos.

El campo teórico de la estética visual será nutrido de la intervención semiótica en el interior del Instituto de Estética de la Pontificia Universidad Católica de Chile con las investigaciones de Radoslav Ivelic, Milan Ivelic, Fidel Sepúlveda y Gaspar Galaz. El Instituto publicará la Revista Aisthesis y habrá trabajos semióticos a partir del número siete.

En las mismas coordenadas históricas Margarita Schultz desarrolla la semiótica musical en el Departamento de Teoría e Historia del Arte de la Universidad de Chile, departamento que a posteriori tendrá un fuerte desarrollo en la semiótica de la estética, con la incorporación de los semióticos Jaime Cordero, María Eugenia Brito (en 1991) y Flu Voionmaa (en 1997).

Pero, el Campo Teórico Hegemónico, en estas coordenadas históricas de 1975 a 1982, será el de la Literatura. La intervención de la semiótica tendrá, en este dominio, una expansión en casi la totalidad de las universidades chilenas. Los centros más importantes que focalizaron el trabajo semiótico fueron:

a) Instituto de Filología Hispánica, Universidad Austral de Chile, Valdivia, bajo la dirección semiótica de Iván Carrasco, Publicaciones: Revista Estudios Filológicos, en la que habrá trabajos semióticos a partir del $\mathrm{n} .^{\circ} 11,1976$.

b) Departamento de Lenguas, Literatura y Comunicación, Universidad de La Frontera, Temuco, Investigadores: María Teresa Poblete, Verónica Contreras, Mabel García, bajo la dirección de Hugo Carrasco, Publicaciones: en Revistas Acta Literaria, Estudios Filológicos y Atenea.

c) Departamento de Español de la Universidad de Concepción, Investigadores: Dieter Oelker, Luis Muñoz, Mario Rodríguez, Mauricio Ostria, bajo la dirección de Roberto Hozven. Publicaciones: Revista Atenea desde el n. ${ }^{\circ} 432 / 1976$ y Revista Acta Literaria a partir del n. ${ }^{\circ}$ 1/1975. 
d) Departamento de Literatura y de Lingüística, Facultad de Filosofía y Humanidades, Universidad de Chile. Investigadores: Luis Waisman, Carmen Foxley, Diamela Eltin, Corina Rosenfeld, en Semiótica Literaria; Gilberto Sánchez, Ambrosio Rabanales, Luis Prieto, en Semiolingüística. Publicaciones: Revista Chilena de Literatura y Revista Lenguas Modernas.

e) Departamento de Idiomas, Universidad de Santiago. Investigadores: Marta Rodríguez, Sergio Pereira, María Eugenia Brito, Rafael del Villar e Ilse Sasso.

f) Departamento de Estudios Generales, Instituto Profesional de Santiago. Investigadores: Guido Vallejos, Sergio Gallardo, María Eugenia Brito y Oscar Aguilera. Publicaciones: Revista Trilogía; a posteriori Universidad Tecnológica Metropolitana.

g) Departamento de Literatura y Ciencias del Lenguaje, Universidad Católica de Valparaíso. Investigadores bajo la dirección de Adolfo de Nordenflycht.

h) Departamento de Artes y Letras, Universidad de la Serena. Investigadores: Manuel Alcides Jofré, Cristián Juan Noemi y Sergio Piñones.

Durante el período 1975-1982 habrá, también, una región epistemológica no hegemónica en el dominio de la semiótica de las comunicaciones. Se trata de una semiótica aplicada a la producción de medios, que abrirá las puertas al tercer período semiótico. No se trata de una institución, sino de investigadores individuales de tres instituciones: Secretariado de Comunicación Social (SEDECOS), Centro Latinoamericano de Educación de Adultos (CLEA) y Centro de Documentación en Comunicación Educativa (CENDOC). Investigadores: María Eugenia Fontecilla y Raymond Colle, quienes a partir de 1980 estarán en la Universidad de Chile (Escuela de Periodismo), y en la Pontificia Universidad Católica de Chile (Escuela de Periodismo), respectivamente.

Lo que es significativo de este período (con la excepción de los dos investigadores anteriormente citados) es que la semiótica se constituye como una disciplina con un lugar institucional en el interior de la literatura y de la estética visual en las universidades chilenas: habrá cátedras, investigadores y publicaciones. Y habrá también un rasgo distintivo: la semiótica llenará una necesidad de los dispositivos teóricos: la necesidad, la carencia es una carencia dentro de la teoría, de la inteligibilidad de los fenómenos, pues no es necesario hacer semiótica para hacer una buena literatura o una buena pintura. 


\section{SEMIÓTICA, REGIONES EPISTEMOLÓGICAS Y RUPTURA: DE 1981 HASTA NUESTROS DÍAS}

Desde 1981, habrá una ruptura epistémica en el campo semiótico. De un lado, una semiótica literaria y visual que se dedica a la inteligibilidad de los fenómenos, cuya necesidad es la de la teoría; y de otro, una semiótica de las comunicaciones que trata de dar respuestas a las demandas de la sociedad. Concretamente es el aparato productivo, la empresa y las organizaciones gubernamentales, quienes originan los problemas que toma a su cargo la semiótica de las comunicaciones. Las preguntas ya no nacen de la ensoñación de la teoría, como era el caso de la semiótica literaria y visual, configurándose así, una ruptura entre dos campos epistémicos.

El Campo Teórico Semiótico de la Estética Visual y de la Literatura, no es más que la expansión cuantitativa (número) y cualitativa (producción) de la región epistémica estatuida en las coordenadas 19751982, descrita precedentemente:

a) Instituto de Filología Hispánica, Universidad Austral de Chile, Valdivia, bajo la dirección semiotica de Iván Carrasco y María Teresa Poblete.

b) Departamento de Lenguas, Literatura y Comunicación, Universidad de La Frontera, Temuco, Investigadores: Orietta Geeregat, Verónica Contreras y Mabel García, bajo la dirección de Hugo Carrasco; y en el Departamento de Filosofía, Hugo Miranda.

c) Departamento de Español de la Universidad de Concepción, Investigadores: Dieter Oelker, Luis Muñoz, Mario Rodríguez, Mauricio Ostria y Mónica Veliz.

d) Departamento de Literatura y de Lingüística, Facultad de Filosofía y Humanidades, Universidad de Chile. Investigadores: Luis Waisman, Carmen Foxley, Diamela Eltin, Corina Rosenfeld, en Semiótica Literaria; Gilberto Sánchez, Ambrosio Rabanales, Luis Prieto, en Semiolingüística.

e) Departamento de Idiomas, Universidad de Santiago. Investigadores: Marta Rodríguez, Claudio Meléndez, Miguel Ángel Farías y Sergio Pereira.

f) Departamento de Estudios Generales, Instituto Profesional de Santiago. Investigadores 1986-1989: Leda Berardi, Sergio Gallardo, María Eugenia Brito, Óscar Aguilera; y desde 1993 hasta 1995 se incorpora Gloria Favi. Hoy denominado Departamento de Estudios Generales, Universidad Tecnológica Metropolitana. Investigaciones actuales bajo la dirección semiótica de Sergio Gallardo.

g) Instituto de Literatura y Ciencias del Lenguaje, Universidad Católica de Valparaíso. Investigadores: Adolfo de Nordenflycht, Erika Cortes y Carlos Díaz Amigo, hasta 1992; desde 1991 Augusto Sarrochi, Sonia Toledo y Adolfo de Nordenflycht. 
h) Departamento de Artes y Letras, Universidad de la Serena. Investigadores: Cristián Noemi, Luis Piñones, Jaime Montes y Mirta Vitar.

i) Departamento de Filosofía, Facultad de Filosofía y Humanidades, Universidad de Chile. Investigadores: Guido Vallejos, Vivian Altman y Federico Schopf.

j) Departamento de Teoría e Historia del Arte, Facultad de Artes, Universidad de Chile. Investigadores: Margarita Schultz, Jaime Cordero, María Eugenia Brito y Flu Voionmaa.

k) Instituto de Estética. Pontificia Universidad Católica de Chile. Investigadores: Radoslav Ivelic, Gaspar Galaz, Fidel Sepúlveda y Milán Ivelic.

1) Instituto de Letras, Pontificia Universidad Católica de Chile. Investigadores: Roberto Hozven, Ana María Burdach, Antonio Arbeas, Luis Flores, Regina Valdéz, Olly Vega y José Luis Samaniego.

m) Departamento de Educación Pre-Escolar, Universidad Metropolitana de Ciencias de la Educación. Investigador: Manuel Alcides Jofré.

n) Departamento de Castellano, Universidad Metropolitana de Ciencias de la Educación. Investigador: Teresa Ayala.

ñ) Departamento de Investigaciones Pedagógicas, Facultad de Filosofía, Universidad de Chile. Investigadores: Sandra Meza y Patricia Soto.

o) Universidad de los Lagos. Investigadores: Eduardo Barraza y Gladys Mora.

p) Universidad Católica Blas Cañas. Departamento de Letras y Educación. Investigadores: Mirta Jara y Grethel Mulhauser.

q) Departamento de Artes de la Representación, Facultad de Artes, Universidad de Chile, Investigadores: John Knukey y Jaime Muñoz.

r) Museo Nacional de Bellas Artes. Investigador: Milán Ivelic.

s) Universidad del Bío-Bío, Facultad de Educación y Humanidades. Investigador: Juan Araya.

Frente a este Campo Teórico de la Semiótica Literaria y de la Estética Visual, surge el Campo Teórico Semiótico de las Comunicaciones como un campo teórico nuevo que entra en ruptura con el anterior en la exacta medida que cambia en 180 grados el edificio epistémico, al tomar como punto de partida el procesamiento de necesidades sociales de la estructura productiva y no las necesidades emergentes de un puro desarrollo de la teoría semiótica. ¿Cuáles son los problemas que plantea la producción masiva de video-música para los comunicadores audiovisuales y en qué puede ayudarles la semiótica? ¿Cuáles son los problemas que plantean a los comunicadores organizacionales la nueva cultura neobarroca o postmoderna y qué les puede aportar la semiótica? ¿Cuáles son los desequilibrios de funcionamiento entre Agencia de Publicidad y Productora de Televisión y qué puede aportar la Semiótica 
de lo Percivible y lo Nombrable? ¿Cuáles son los problemas que la construcción de imágenes corporativas plantea a los publicistas y comunicadores organizacionales un mundo hipersegmentado y heterogéneo culturalmente y qué puede aportar la semiótica? ¿Cuáles son los problemas que el cambio de una cultura masiva a una hipersegmentada plantea a los publicistas y qué puede aportar la semiótica? Las preguntas precedentes ejemplifican este vuelco epistémico, donde la intervención semiótica es hecha a partir de una pregunta cuyo origen no es la pragmática semiótica sino un hacer comunicacional concreto, producto de un hacer profesional en Empresas Concretas y Organizaciones Gubernamentales, lo que se refleja en publicaciones de esta línea epistémica: Rafael del Villar, 1992, 1996, 1997; Raymond Colle, 1993; Manuel Alcides Jofré, 1995; Francisco Fabres, 1995, Julio Reyes, 1995; Erika Cortés, 1996; Leda Berardi, 1996, 1997; Sonia Montesino, 1996; Malva Sánchez, 1996; Grabiela Aliste, 1996; Hugo Carrasco, 1997; Elisa Montesinos, Marcela Ponce, Ana María Delgado, e Ibi Panger, 1997; Nancy Luco, 1997; Francisco Alderete, 1997; Georgina Mora, 1997, entre otros.

Esta nueva semiótica tiene una realidad de funcionamiento en las universidades chilenas y en el campo profesional a través de instituciones que plantean la necesidad de asesorías semióticas y/o la realización de mini-estudios empíricos. Así, aparecen semióticas vinculadas a estructuras productivas comunicacionales en:

- Servicio Agrícola Ganadero (SAG, bajo la dirección de Malva Sánchez),

-Corporación Nacional Forestal (CONAF, bajo la dirección de Eliana Chong).

-Instituciones Previsionales (PROVIDA, bajo la dirección de Gabriela Aliste).

- Consejo Nacional de Televisión (Víctor Fajnzylber, Alejandra Ram, en el interior de la Unidad de Estudios Cualitativos, dirigida por Carlos Catalán).

-Banco de Santiago (bajo la dirección de Julio Reyes).

- Servicio Nacional de la Mujer (bajo la dirección de Carolina López y Marcela Ponce).

- Secretaría Nacional de Gobierno (Unidad de Estudios Cualitativos, bajo la dirección de Francisco Alderete).

- Políticas Públicas y Salud (Subconjunto del Programa Intergubernamental de Políticas de Población, a cargo de Leda Berardi).

-Productoras Audiovisuales Concretas (Videogram, bajo la dirección de Francisco Fabres). 
-Empresas de Publicidad (por ejemplo, Adimark, Unidad de Estudios Cualitativos, bajo la dirección de Carlos Vila; entre otras).

-Municipalidades (Pudahuel, bajo la dirección de Francisco Alderete); etc.

En las universidades esta nueva semiótica comienza a producirse alrededor:

a) De las Escuelas de Periodismo y Master en Comunicación de la Universidad de Chile Investigadores: María Eugenia Fontecilla, hasta 1993; Rafael del Villar, Leda Berardi, Gloria Favi, desde 1991 hasta hoy; y Óscar Aguilera desde 1986 hasta 1996.

b) De la Escuela de Periodismo de la Pontificia Universidad Católica de Chile, bajo la dirección de Raymond Colle.

c) De la Escuela de Periodismo de la Universidad de Santiago de Chile. Investigadores: Claudio Meléndez y Carlos Mejías, desde marzo de 1995 hasta hoy.

d) De la Carrera de Publicidad de la Universidad de Santiago de Chile. Investigadores: Rafael del Villar desde 1981 a 1991 y Jorge Brower desde 1992 hasta hoy.

El desarrollo del Campo Teórico Semiótico de las Comunicaciones está directamente ligado a la expansión de las comunicaciones en la sociedad chilena. La economía de este país se encuentra inserta en 1981 en los procesos de globalización económica mundial, con una hiperinterdependencia e hiperespecialización de la actividad productiva, en el marco de mercados ultracompetitivos. Así Chile exporta materias primas a los mercados americanos, europeos, latinoamericanos y asiáticos; a su vez que empresas de servicios, sobre todo, a América Latina. Esto hace necesario implementar estrategias comunicativas, y la publicidad y el diseño gráfico tienen un lugar privilegiado. Por el bajo costo comparativo, y la estructura tecnológica del país, pronto Chile se transforma en exportador de publicidad y diseño, lo que incrementa más aún la expansión de dichas disciplinas. Es por ello que el proceso de globalización y diversificación cultural de la década de los 90 se encuentra, en la realidad chilena, con un modelo de libre mercado que incentiva el dominio de las comunicaciones y que ya había desplazado el centro de la producción televisiva a productoras independientes, que vendían los programas audiovisuales a los cuatro canales de televisión abierta existentes en 1989: Televisión Nacional de Chile (dos frecuencias: 7 y 9), Corporación de Televisión Univer- 
sidad Católica, Universidad de Chile Televisión y Universidad Católica de Valparaíso Televisión. Este proceso expansivo hace que en Santiago, en 1997, nos encontremos con 8 canales de Televisión Abierta producidos en Chile, y 16 canales de T.V. Cable de Producción Nacional (en una oferta televisiva total de 74 canales), lo que implica la emergencia de una cultura audiovisual diversificada y un cambio en el consumo de medios, que plantea nuevas preguntas y nuevos desafíos para los comunicadores, pues se pasa de un consumidor masivo a uno hipersegmentado, con todas las carencias analíticas que ello implica. De ahí, la importancia de las comunicaciones y de la semiótica de las comunicaciones, que se ve como un aporte importante a la inteligibilidad y producción audiovisual. Por otra parte, el abaratamiento de los costos de los sistemas de impresión, y de los sistemas de producción audiovisual, retroalimenta la generación de micromedios; esto es, circuitos comunicacionales en el interior de empresas, que quieren reflejar a sus integrantes una imagen corporativa que los identifique, al mismo tiempo que optimizar sus flujos comunicacionales formales e informales; lo que no puede realizarse a través del saber de las relaciones públicas, sino que a partir del saber transdisciplinario de la comunicación organizacional: se requiere detectar los climas organizacionales, los flujos comunicativos, las catástrofes de funcionamiento de la organización, lo que se detecta a través de los textos generados por la organización y el procesamiento semiótico de los mundos posibles de los receptores de la estrategia comunicativa; es clara, entonces, la necesidad de la intervención semiótica junto al saber de la psicología y la sociología organizacional y de las relaciones industriales. De ahí, la importancia de las comunicaciones y de la semiótica de la comunicación organizacional, como un subconjunto disciplinario emergente.

Esta importancia que adquiere el saber comunicacional, y el saber específico de la semiótica de las comunicaciones, se ve reflejada en la existencia de cátedras de semiótica en las carreras de periodismo y de publicidad en las universidades descritas precedentemente: Universidad de Chile, Pontificia Universidad Católica de Chile y Universidad de Santiago de Chile.

Sin embargo, en la década analizada se producen, además, profundas transformaciones en la estructura productiva de la generación del saber, habiendo, en la Educación Superior, dos cambios importantes: la culminación de un proceso de descentralización (originado a partir de 1974) y la emergencia de universidades privadas (que coincide 
con las coordenadas del Campo Teórico Semiótico de 1981-hasta hoy).

En Chile existían dos grandes universidades: la Universidad de Chile y la Pontificia Universidad Católica de Chile (y aún hoy, son las dos, los principales centros de enseñanza superior), que abarcaban todo el territorio nacional, teniendo centros en cada región del país. Junto a ellas existían cuatro universidades más pequeñas en Santiago (la Universidad Técnica del Estado, hoy Universidad de Santiago de Chile), en Concepción (la Universidad de Concepción) y en Valparaíso (la Universidad Católica de Valparaíso y la Universidad Técnica Federico Santa María). Es ése el contexto universitario en que se insertaba la primera semiótica datada en las coordenadas de 1969. El proceso de descentralización significó crear universidades regionales independientes con cada una de las sedes de ambas universidades centrales (la Universidad de Chile y la Pontificia Universidad Católica) y, a su vez, crear universidades para áreas específicas del saber. Así, la Universidad de Chile (sede Santiago) dio origen a dos universidades independientes: la Universidad Metropolitana de Ciencias de la Educación y la Universidad Tecnológica Metropolitana, referidas respectivamente a la formación de profesores y a la formación de carreras técnicas como trabajo social, construcción civil, bibliotecología, entre otras.

La segunda semiótica descrita en su campo teórico (1975-1982) corresponde a la existencia de universidades regionales independientes. El período de la tercera semiótica (a partir de 1981), se encuentra ya con el proceso de descentralización terminado, a través de la existencia de universidades en las dos áreas del saber descritas. La finalización del proceso de descentralización de las universidades chilenas implicó todo un proceso de reestructuración de los investigadores del campo de la semiótica, desde el punto de vista de su ligazón institucional, como asimismo la generación de nuevos grupos de investigación en nuestro campo disciplinario.

La finalización del proceso de descentralización de las universidades chilenas se unió a otro proceso de transformación: la emergencia de educación superior privada, lo que tendrá un marco jurídico a partir de 1981. La educación superior privada se diferenció en Centros de Formación Técnica, Institutos Profesionales y Universidades. Si en el período de la primera semiótica había seis universidades de servicio público financiadas por el estado, y si en el período de la segunda semiótica existían veinticuatro universidades de servicio público 
financiadas por el estado, producto del proceso de descentralización de la actividad universitaria señalado; ahora, en 1997, habrá sesenta y cuatro universidades, cuarenta de las cuales son privadas. Los Institutos Profesionales tendrán, también, un fuerte crecimiento, teniendo hoy, una oferta de 109 Institutos Profesionales Privados a lo largo del país.

Ahora bien, en Santiago hay veinte y ocho universidades privadas, y trece tienen carreras del ámbito de la comunicación, casi el 50\%. Lo mismo ocurre en los Institutos Profesionales a lo largo del país: de los 110 existentes, 61 tienen carreras vinculadas a la comunicación, lo que significa que un $55 \%$ de la oferta de educación superior se desarrolla en dicho ámbito. Periodismo, Publicidad, Comunicación Audiovisual, Diseño Gráfico, Danza, Tecnología del Sonido, Comunicación Escénica, Comunicación Multimedial, entre otras, se constituyen en una oferta pertinente para instituciones de educación privadas que deben competir con los sistemas tradicionales de educación superior. De ahí que encuentren en la satisfacción de las demandas del mercado (y del prestigio de las comunicaciones en la sociedad chilena de la última década) una fuente de distintividad. Una característica típica de las instituciones privadas de enseñanza, en el proceso chileno, es, entonces, la ligazón universidad-empresa. La rentabilidad presupuesta de las comunicaciones hace necesario implementar una institucionalización de la enseñanza de las comunicaciones, que encuentra su modelo en dicha relación, pero a su vez, en el proceso de acumulación tradicional del saber de las universidades tradicionales de servicio público, pues no pueden partir de un punto cero de acumulación del saber. De ahí, que la presencia de la semiótica en la Universidad de Chile (Escuela de Periodismo), en la Universidad de Santiago (Carrera de Publicidad) y en la Pontificia Universidad Católica de Chile (Escuela de Periodismo) tenga una buena acogida en las instituciones privadas de enseñanza superior. Lo que se retroalimentó con el éxito de la primera institución superior privada referida sólo al ámbito de las comunicaciones, el Instituto Superior de Artes y Ciencias de la Comunicación (IACC, posteriormente transformado en la primera universidad privada sólo referida al ámbito de las comunicaciones, UNIACC), que incluyó a la semiótica como disciplina obligatoria en todas sus carreras, en una visión postmoderna apropiada no sólo a la inteligibilidad de mensajes sino a la producción audiovisual, lo que implicó la emergencia de carreras similares en otra Universidades e Institutos de Enseñanza Superior, donde la semiótica tiene, en la mayoría, a lo menos, presencia. 
Así hoy, en 1997, debemos agregar a la lista de instancias institucionales de investigación semiótica en comunicación los centros de docencia y/o investigación privados. Algunos de los principales son:

e) Universidad de Artes Ciencias y Comunicación UNIACC. DocentesInvestigadores: Guadalupe Álvarez, Gustavo Cárdenas, Mario Osses, Felipe Alarcón, Andrés Grimblatt y Rafael del Villar.

f) Universidad del Pacífico. Investigadores bajo la dirección de Erika Cortez y Gloria Favi.

g) Universidad Diego Portales. Docentes-Investigadores: Eduardo Lawrence, Alejandra Ram y Jorge Brower.

h) Universidad Arcis. Docentes-Investigadores: Luis Torres, Raúl Bendezu, Vicente Sisto y Eduardo Román.

i) Universidad de Viña del Mar. Investigadores bajo la dirección de Eduardo Román.

j) Universidad Bolivariana. Investigadores bajo la dirección de Nancy Luco.

k) Universidad Católica Blas Cañas. Departamento de Ingeniería en Administración. Investigadores bajo la dirección de Carolina Olmos.

1) Universidad Tecnológica Vicente Pérez Rosales. Investigadores bajo la dirección de Pavella Coppola.

m) Universidad Internacional SEK. Docente-Investigador: Augusto Cabeza.

n) Universidad Finis Terrae. Docencia.-Investigación: Eduardo Guerrero.

o) Instituto Profesional DUOC-Pontificia Universidad Católica de Chile. Docentes-Investigadores bajo la dirección de Erika Cortés y Loreto Lamas.

p) Instituto Profesional INACAP. Docencia-Investigación a cargo de Andrea Gougain y Carolina Olmos.

q) Escuela de Comunicación. Docentes-Investigadores bajo la dirección de María Eugenia Fontecilla, Salvador Benadava, Eduardo Lawrence y Carlos Villa.

r) Instituto Profesional Arcos. Docentes-Investigadores bajo la dirección de Vera Carneiro, Nelson Olagaray, Demetrio Sijas y Jaime Muñoz.

s) Instituto Profesional Procom. Docencia: Víctor Hugo Muñoz.

t) Instituto Profesional AIEP. Docencia a cargo de Fulvio Cefferoni

A esta lista debemos agregar las universidades regionales tradicionales de servicio público que insertan la semiótica como subconjunto básico en su propuesta investigadora y curricular: 
u) Escuela de Periodismo y Programa de Máster en Comunicación, Universidad de la Frontera, Temuco. Investigadores: Orietta Geeregat, Verónica Contreras, Mabel García, bajo la dirección de Hugo Carrasco.

v) Universidad Católica del Norte, Escuela de Periodismo, Antofagasta. Docencia-Investigación bajo la dirección de Georgina Mora e Irene Ramallo.

w) Universidad del Bío-Bío, Departamento de Comunicación Visual, Chillán. Docencia-Investigación a cargo de Norman Ahumada Gallardo.

A medio camino, entre una semiótica que busca sus preguntas en la teoría semiótica general (la semiótica de la literatura y la semiótica de la estética visual) y la que perfila sus interrogantes a partir de la pragmática comunicacional de las empresas y el Estado (la semiótica de las comunicaciones) se genera paulatinamente, desde las coordenadas de 1991 en adelante, una tercera región epistemológica: la de la semiótica en antropología y sociología. Dicha región emergente se nutre por una parte de las debilidades del análisis de contenido cuantitativo, y por otra, de las necesidades inteligibilizadoras que plantea un mundo hipersegmentado y contradictorio en el ámbito de la cultura. De ahí, las temáticas que constituyen los subconjuntos mismos en que se insertan:

a) Programa Interdisciplinario de Estudios de Género, Departamento de Antropología, Facultad de Ciencias Sociales, Universidad de Chile. Docencia-Investigación dirigida por Sonia Montecino y Loreto Rebolledo, desde 1991 hasta hoy.

b) Unidad Análisis de Contenido y Semiótica, Departamento de Sociología, Universidad de Chile. Docencia-Investigación bajo la dirección de Manuel Canales, desde 1993 hasta hoy.

c) Programa de Estudios de Género, Facultad de Filosofía y Humanidades, Universidad de Chile. Docencia-Investigación dirigida por Kemy Oyarzún, desde 1995 hasta hoy.

d) Unidad Análisis de Contenido y Semiótica, Instituto de Sociología, Pontificia Universidad Católica de Chile, subconjunto de la Unidad de Análisis Cualitativo, dirigida por Carlos Catalán: María Dolores Sauza, Alejandra Ram y Víctor Fajnzylber, desde 1996 a hoy.

e) Unidad de Etnolingüística y Semiótica, Departamento de Antropología, Facultad de Ciencias Sociales, Universidad de Chile. Investigación bajo la dirección de Oscar Aguilera, desde 1997 hasta hoy.

Esta nueva región epistémica en formación retroalimenta cuantitativamente y cualitativamente el campo semiótico ya expandido en la institucionalización de la educación superior chilena, a través de los 
procesos de descentralización y emergencia de institutos profesionales y universidades privadas ya descritos, situación a la que han contribuido las demandas de la estructura productiva al mirar semiótico.

Lo más significativo, entonces, en la semiótica chilena del tercer período, es esta expansión del mercado ocupacional de semióticos y/o semiólogos, más allá de la dinámica interna de la disciplina. El mercado ocupacional de la semiótica no crece por un desarrollo institucional de la semiótica como ciencia, sino por los procesos sociales: a) de descentralización de la actividad universitaria; b) de tornarse importante para la sociedad (la empresa y los organismos gubernamentales) el quehacer publicitario, el diseño gráfico, y la industria audiovisual, requiriéndose demandas concretas a la semiótica; c) de profundas transformaciones culturales en el consumo de medios, producto de la globalización y diversificación de las comunicaciones, que hacen necesario el desarrollo de técnicas cualitativas de procesamiento de la información, las que encuentran en la semiótica una vía analítica inteligibilizadora; y d) de emergencia de educación superior privada que hacen suyas las nuevas demandas de la estructura productiva de la sociedad, a su vez que las liga al proceso de acumulación del saber de las universidades de servicio público, donde la semiótica tenía ya un lugar privilegiado. Esto significa que las necesidades de mini-investigaciones, y de docencia, en el campo de la semiótica, crecen en forma geométrica, y es mayor que la misma generación de semióticos y/o semiólogos en el interior de las formas institucionales tradicionales de formación académica. Es éste el rasgo más relevante de la institucionalización de la semiótica como disciplina en el Chile de la actualidad. De hecho este rasgo estaba presente ya en la primera semiótica, en sus orígenes, pues eran las necesidades de la lucha ideológica de la sociedad las que le daban a la semiótica un lugar; hoy es la sociedad, más allá de ella misma como disciplina, quien le da un lugar.

\section{INSTITUCIONALIZACIÓN DE LA SEMIÓTICA Y LA ASOCIACIÓN CHILENA DE SEMIÓTICA}

En 1994 se creó la Asociación Chilena de Semiótica, organización que agrupa a quienes trabajan en la disciplina, con personalidad jurídica desde 1996. Los principios que estructuran a la Asociación están en la articulación polidialógica de tres ejes: a) la representación de las tres 
regiones del país: el norte, el sur, y el centro; b) la representación de las diferentes regiones epistémicas que cristalizan el hacer semiótico: la sociología y la antropología, las comunicaciones, y finalmente, la literatura y la estética visual; y c) la creación de instancias de interconexión entre la actividad académica y la actividad productiva. Estos principios se ven reflejados en los Estatutos de la Organización, como asimismo en el primer comité directivo elegido para el período 1994-1995:

— Rafael del Villar Muñoz, Presidente (Comunicaciones, Universidad de Chile).

- Leda Berardi y Carlos Vila, Coordinadores de la Presidencia (Comunicaciones, Universidad de Chile; y Comunicaciones Empresa de Marketing Adimarck, respectivamente).

-Vicepresidentes: Flu Voionmaa (Estética Visual, Universidad Finis Terra), Sergio Pereira (Literatura, Universidad de Santiago), Hugo Carrasco (Literatura, Universidad de la Frontera), Guido Vallejos (Filosofía, Universidad de Chile), Vera Carneiro (Comunicación, Instituto Profesional Arcos) y Elena Torres (Corporación Chilena de Mujeres de Negocio y Profesionales).

-Secretaria General: Mirta Jara (Estética Visual, Universidad Católica Blas Cañas).

-Secretario General Adjunto: Julio Reyes (Comunicaciones, Banco de Santiago).

- Tesorero: Gustavo Cárdenas (Comunicaciones, Universidad Uniacc).

-Editor-Publicaciones: Óscar Aguilera (Antropología, Universidad de Chile).

En mayo de 1996 se realizó el Primer Encuentro Chileno de Semiótica, cuyo objetivo fue establecer un diagnóstico del hacer semiótico en Chile, a través de la presentación de trabajos de investigación en curso (40 ponencias), organizado por el Departamento de Ciencias y Técnicas de la Comunicación, de la Universidad de Chile y la Asociación Chilena de Semiótica. Se celebró allí, también, la Segunda Asamblea General de la Asociación, donde se reafirmaron los principios centrales que rigen a la Asociación, y se eligió su segundo comité directivo (período 1996-1999), que mantiene los mismos ejes anteriores de interconexión polidialógica de los tres principios rectores de la organización:

-Presidente: Rafael del Villar (Comunicación, Universidad de Chile).

- Coordinadores de la Presidencia: Leda Berardi (Comunicación, Universidad de Chile) y Carlos Vila (Comunicaciones, Empresa de Marketing Adimarck). 
-Vice-Presidentes: Hugo Carrasco (Comunicación, Universidad de la Frontera), Iván Carrasco (Literatura, Universidad Austral); Radoslav Ivelic (Estética Visual, Pontificia Universidad Católica de Chile); Luis Piñones (Literatura, Universidad de la Serena) y Margarita Schultz (Estética, Universidad de Chile).

-Secretaria General: Mirta Jara (Estética, Universidad Católica Blas Cañas).

-Secretario General Adjunto: Julio Reyes (Comunicaciones, Banco de Santiago).

-Tesorera: Grethel Mulhauser (Estética, Universidad Católica Blas Cañas).

-Editor-Publicaciones: Óscar Aguilera (Antropología, Universidad de Chile).

Desde 1996 el Departamento de Ciencias y Técnicas de la Comunicación de la Universidad de Chile y la Asociación Chilena de Semiótica publican la Revista Chilena de Semiótica, a través de Internet (http://rehue.csociales.uchile.cl/ rehuehome/ facultad/ publicaciones/ semiotica/ semiotica2/ index. html.), habiéndose publicado dos números. El primero toma la temática del primer encuentro, referido a dar cuenta de las investigaciones en curso en diferentes ámbitos nacionales, y el segundo tiene como objetivo dar una descripción del carácter contradictorio y polidialógico de la cultura chilena actual. La revista se ha transformado en una instancia de comunicación de los distintos haceres semióticos del país, como asimismo del cono sur de América Latina, recibiéndose colaboraciones de Argentina, México, Perú y Bolivia.

La Asociación Chilena de Semiótica tiene 218 miembros a lo largo del país, 120 son académicos de las universidades chilenas, 48 son estudiantes que participan en las actividades de la Asociación, y 50 son miembros individuales profesionales que desarrollan la semiótica aplicada a tareas productivas. Composición que cristaliza los principios constitutivos de la semiótica en el Chile de hoy.

\section{Referencias bibliográficas}

AgUileRA, ÓsCAR (1985). «Un documento rescatado para la historia de la investigación lingüística Kawésqar (alacalufe)». Trilogía 5.9. Santiago: Ed. Instituto Profesional de Santiago. 
- (1997). «La expresión del espacio Kawésqar». Revista Etno 1. Santiago: Internet: Http:// rehue. csociales. uchile. cl/ rehuehome/ facultad/ publicaciones/ Ethno/ index. html. Facultad de Ciencias Sociales, Universidad de Chile.

- (1997). «La expresión del tiempo en Kawésqar». Revista Onoma 1. Santiago: Ediciones Instituto de Letras, Pontificia Universidad Católica de Chile.

- (1998). «El tema de la muerte en la literatura oral Kawésqar. Anales de la Universidad de Chile, Primer semestre. Santiago: Ediciones Universidad de Chile.

ALDERETE, FRANCISCO (1997). Investigación de cultura en las comunicaciones de la organización. Santiago: Tesis Magister en Comunicación, Facultad de Ciencias Sociales, Universidad de Chile.

Aliste, GABRIEla (1996). Pasos para evaluar la comunicación interna en una empresa de servicios previsionales. Santiago: Tesis Magister en Comunicación Social, Facultad de Ciencias Sociales, Universidad de Chile.

BERARDI, LEDA (1996). «Legitimidad y discuso presidencial. Un análisis de los discursos de los Presidentes Eduardo Frei Montalva y Eduardo Frei Ruiz-Tagle». Revista Chilena de Semiótica 1. Santiago, Internet: http://rehue. csociales. uchile. cl/ rehuehome/ facultad/ publicaciones/ semiotica/ semiotica2/ index. html. Ed. Facultad de Ciencias Sociales, Universidad de Chile.

- (1997 a). «Estrategias discursivas y legitimidad». En L. Guzmán (ed.), Exploraciones en psicología política I. Santiago: Ediciones Universidad Diego Portales.

- (1997 b). «Características de los discursos de los Presidentes Eduardo Frei Montalva y Eduardo Frei Ruiz-Tagle». En A. Bolivar, y P. Bentivoglio (eds), Actas del primer coloqui latinoamericano de analistas del discurso. Caracas: Ediciones Universidad Central de Venezuela.

BRITO, MARÍA EUGENIA (1988). «El quiasmo: figura que perfila y consagra la gesta de la conquista en Chile». Anuario del Departamento de Humanidades. Santiago, Ediciones del Instituto Profesional de Santiago

- (1990). Campos minados (literatura post-golpe en Chile). Santiago: Editorial Cuarto Propio.

- (1995). «Teresa de Rosario Orrego, una lectura política del amor». Revista de Crítica Cultural II Semestre

- (1996). «Homologías y continuidades: la novela del siglo XIX y principios del XX: el texto colonial y poscolonial». Revista Chilena de Semiótica 2. Santiago, Internet: http: // rehue. csociales. uchile. cl/ rehuehome/ facultad/ publicaciones/ semiotica/ semiotica2/ index. html. Ed. Facultad de Ciencias Sociales, Universidad de Chile.

BROWER, JORGE (1996). «Semiología del otro hombre como manifestación de Dios más allá del sistema de significación ontometafísico». Revista 
Chilena de Semiótica 1. Santiago, Internet: http: // rehue. csociales. uchile. $\mathrm{cl} /$ rehuehome/ facultad/ publicaciones/ semiotica/ semiotica2/ index. html. Ed. Facultad de Ciencias Sociales, Universidad de Chile.

Carrasco, Hugo (1978). "Drama en el drama en Nuestra Natacha». Estudios Filológicos 13. Valdivia: Ed. Universidad Austral.

- (1980). «El problema del destinatario en Las Meninas, de Buero Vallejo». Estudios Filológicos 15. Valdivia: Ed. Universidad Austral.

- (1982). «Las narraciones concurrentes en La isla y los demonios». Estudios Filológicos 17. Valdivia: Ed. Universidad Austral.

- (1983). «Sobre la noción de relato oral mapuche». Actas II Seminario Nacional de Estudios Literarios. Santiago: Ed. Universidad de Santiago.

- (1984). «Trentren y Kaikai: ¿mito de origen en la cultura mapuche? En Actas III Seminario Nacional de Estudios Literarios. Temuco: Ed. Universidad de la Frontera.

- (1985). «Sistema mítico y relato oral mapuche». Estudios Filológicos 20. Valdivia: Ed. Universidad Austral.

- (1988). «Un mito mapuche anterior a Trentren y Kaikai». Estudios Filológicos 23. Valdivia: Ed. Universidad Austral.

- (1997). «El viaje al otro mundo en la gramática mítica mapuche». Revista Chilena de Semiótica 2. Santiago, Internet: http: // rehue. csociales. uchile. cl/ rehuehome/ facultad/ publicaciones/ semiotica/ semiotica2/ index. html. Ed. Facultad de Ciencias Sociales, Universidad de Chile.

CARRASCO, IVÁN (1978). «El antipoema de Parra: una escritura transgresora». Estudios Filológicos 13. Valdivia: Ed. Universidad Austral.

- (1979). «Dos discursos complementarios: las dedicatorias y las notas». Estudios Filológicos 14. Valdivia: Ed. Universidad Austral.

- (1980). «Naturaleza y función de las acotaciones (a propósito de Buero Vallejo)». Estudios Filológicos 15. Valdivia: Ed. Universidad Austral.

- (1981). «En torno a la producción verbal artística de los mapuches». Estudios Filológicos 16. Valdivia: Ed. Universidad Austral.

- (1988). «Antipoesía y neovanguardia». Estudios Filológicos 23. Valdivia: Ed. Universidad Austral.

- (1989). «El proyecto poético de Raúl Zurita». Estudios Filológicos 24. Valdivia: Ed. Universidad Austral.

- (1996). «Discurso metatextual e interculturalidad. Un ejemplo mapuche». Revista Chilena de Semiótica 1. Santiago, Internet: http: // rehue. csociales. uchile. cl/ rehuehome/ facultad/ publicaciones/ semiotica/ semiotica2/ index. html. Ed. Facultad de Ciencias Sociales, Universidad de Chile.

COLLE, RAYMOND (1975). Estructura de la red de comunicación en las escuelas radiofónicas. Santiago: Ed. SEDECOS, mimeografiado. 
- (1977). El archivo visual. Santiago: Ediciones Paulinas.

- (1977). El sonorama. Santiago: Ed. Paulinas.

- (1978). Manejo de información por vía gráfica en investigaciones socio- educativas. Osorno, Ed. ISIFREDER.

- (1982). El lenguaje de la imagen: iniciación a la semiótica visual. Santiago: Ed. CENCOSEP.

- (1993). Iniciación al lenguaje de la imagen. Santiago: Ediciones Universidad Católica de Chile.

CONTRERAS, VeróniCa (1984). «Casa de Campo o la consciencia de escritura». En Actas III Seminario Nacional de Estudios Literarios. Temuco: Ed. Universidad de La Frontera.

CORDERO, JAIME (1972). Sistema comparativo de las proposiciones concesivas. Grenoble: Archives Documentaires Université de Grenoble.

- (1976). El recuerdo del olvido: por una memoria afectiva. Santiago: Ed. Universidad de Santiago.

- (1978). «Enseignements et aprendissage d'L2». Revista de Lenguas modernas 5. Santiago: Ed. Facultad de Filosofía, Universidad de Chile.

- (1990). «El relativismo en lingüística». En Cordero y Estrella (eds.). Viejas y nuevas fronteras de la ciencia. Santiago: Editorial Universitaria.

- (1993). «La teoría del caos». En Revista del Instituto de Ingenieros de Chile. Santiago: Ediciones Facultad de Ingeniería, Universidad de Chile.

CORTÉs, ERIKA (1990). «Envolvimiento: crisis de identidad en los personajes de El obsceno pájaro de la noche». Signos 23/ I y II Semestre. Valparaíso: Ediciones Universidad Católica de Valparaíso.

- (1991). «Este Domingo. Nostalgia del paraíso perdido como imagen de lo auténtico». Signos 24/ II Semestre. Valparaíso: Ediciones Universidad Católica de Valparaíso.

- (1992). «Interpretación histórica del héroe en dos obras dramáticas: Ayayema y Lautaro». En Actas Séptimo Congreso Nacional Sociedad Chilena de Estudios Literarios. Literaturas hispánicas de América en el quingentésimoaniversario del descubrimiento, Valparaíso: Ediciones Universidad de Playa Ancha de Ciencias de la Educación.

- (1994). «El escenario: un símbolo postmodernista». En Actas Octavo Seminario Internacional de Estudios Literarios. Osorno: Ediciones Universidad de los Lagos.

- (1996). «Funcionalidad y aplicaciones de la semiótica teatral». Revista Chilena de Semiótica, 1. Santiago, Internet: http: // rehue. csociales. uchile. cl/ rehuehome/ facultad/ publicaciones/ semiotica/ semiotica2/ index. html. Ed. Facultad de Ciencias Sociales, Universidad de Chile.

DE NORDENFLYCHT, ADOLFo (1984). «Intertextualidad en abismo: a propósito de «El poeta de Campo» de Jorge Teillier». En Actas III Seminario Nacional de Estudios Literarios, Temuco: Ed. Universidad de la Frontera. 
Del Villar, Rafael (1971a). Mensaje (forma y contenido) e ideología. Valparaíso Publicaciones Previas ICSD/UCV.

- (1971b). La construcción estructuralista de los mensajes. Valparaíso Publicaciones Previas ICSD/UCV.

- (1971c). La construcción estructuralista marxista de los mensajes. Valparaíso Publicaciones Previas ICSD/UCV.

- (1972). «De cómo tanto la forma visual como su contenido son expresión de Ideología». En Revista Primer Plano 1, Valparaíso: Ediciones Universitarias de Valparaíso.

- (1981). Semiología de la imagen visual publicitaria. Santiago: Mimeo. Central Apuntes, Escuela Tecnológica/ Universidad de Santiago.

- (1983). «Consideraciones comparativas sobre la semiótica narrativa, la semántica antropológica y el análisis textual en el análisis literario». En Actas II Seminario Nacional de Estudios Literarios. Santiago: Ed. Universidad de Santiago.

- (1984). «La narratividad del cine contemporáneo y su transgresión de la narratividad clásica: consideraciones para su inteligibilización». En Actas Tercer Seminario Nacional de Estudios Literarios». Temuco: Ed. Universidad de la Frontera.

- (1989). «Una herramienta semiótica para la evaluación de un vídeo educativo en su proceso de gestación/producción». Korean Educational Development Review 2/1989.

- (1992). «La pragmatique d'un modèle sémiotique construit pour l'évaluation des vidéo-clips dans leur procés de géstation/ production». En Deledalle (ed), L' Homme et ses sigres. Berlín: Editorial Mouton de Gruyter.

- (1992). «Una herramienta analítica audiovisual aplicada a la transmisión de la identidad Latinoamericana». En Barbero, Sóliz, Núñez (eds.), En torno a la Identidad Latinoamericana. México: Ed. Felafacs.

- (1996). «Cultura de la imagen contemporánea y producción televisiva». Tercer milenio 1. Antofagasta: Ediciones Universidad Católica del Norte.

- (1996). «Proyecto video institucional Finzatel» (en colaboración con Carol Parker y Leslie O'ryen). Revista Chilena de Semiótica 1. Santiago, Internet: http: // rehue. csociales. uchile. cl/ rehuehome/ facultad/ publicaciones/ semiotica/ semiotica2/ index. html. Ed. Facultad de Ciencias Sociales, Universidad de Chile.

- (1997). «Sémiotique au Chili d'ajourd'hui: histoire, ruptures et champ théorique». En Rauch y Carr (eds.), Semiotics around the world: synthesis in diversity. Berlín: Ed. Mouton de Gruyter.

- (1977). «Le positionnement pluriel: le travail sur l'ambigüité du texte». En Rauch y Carr (eds.), Semiotics around the world: synthesis in diversity. Berlín: Ed. Mouton de Gruyter.

- (1977). Trayectos en semiótica fílmica televisiva. Santiago: Dolmen Ediciones. 
- (1977). «La complementariedad de Lévi-Strauss, Kristeva y PetitotCocorda, en la inteligibilización del universo semántico y pulsional del texto lingüístico». Revista Chilena de Semiótica 2. Santiago, Internet: http: // rehue. csociales. uchile. cl/ rehuehome/ facultad/ publicaciones/ semiotica/ semiotica2/ index. html. Ed. Facultad de Ciencias Sociales, Universidad de Chile.

Del Villar, Rafael y ConTreras, EduARDo (1971). Comunicación e ideología: objeto, teoría y metodología. Valparaíso: Ediciones Previas ICSD/ UCV.

EChEVERRÍA, RAFAel (1990). Ontología del lenguaje. Santiago: Dolmen Ediciones.

FAJNZYLBER, VíctoR (1997). El lenguaje de los dibujos: una experiencia en semiótica animada. Santiago: Tesis de Grado, Taller de Comunicación de Masas, Instituto de Sociología, Pontificia Universidad Católica de Chile.

FonTECILla, MARIA EUGENIA (1975). La radio cultural. Santiago: Ed. SEDECOS, mimeografiado.

- (1976). Medios masivos y educación de adultos. Santiago: Ed. CENDOC, mimeografiado.

- (1980). Los procesos de alfabetización, un enfoque comunicacional. Santiago: Ed. CLEA, mimeografiado.

- (1981). Semiótica de la Publicidad, Semiótica de la Comunicación Organizacional. Santiago: Programa Magistratura en Comunicación, Universidad de Chile, mimeografiado.

FOXLEY, CARMEN (1984). «La propuesta autorreflexiva de «Anteparaíso». En Actas III Seminario Nacional de Estudios Literarios. Temuco: Ed. Univ. de la Frontera.

- (1985). «El discurso de Nicanor Parra y las presuposiciones». Estudios Filológicos 20. Valdivia: Ed. Universidad Austral.

Greimas, Algirdad Julien (1966). Sémantique structurale. París: Ed. Larousse.

Hozven, Roberto (1976). «El modelo genético de Lévi-Strauss». Acta Literaria 3. Concepción: Ed. Universidad de Concepción.

- (1978). «Un modelo estructural y tres relatos orales chilenos». Estudios Filológicos 13. Valdivia: Ediciones Universidad Austral.

- (1979). «El estructuralismo literario francés. Santiago: Ed. Fac. Ciencias Físicas y Matemáticas, Universidad de Chile.

IVElic, Milán (1973). Curso de Estética General. Santiago: Ed. del Pacífico.

- (1975-76). «Pintura y Percepción». Aisthesis 9. Santiago: Ed. Universidad Católica.

IVELIC, RADOSLAV (1975-1976). «La inefabilidad pictórica». Aisthesis 9. Santiago: Ed. Universidad Católica.

- (1996). «Semiótica y estética. Estructuras semióticas del arte». Revista Chilena de Semiótica, 1. Santiago, Internet: http: // rehue. csociales. uchile. $\mathrm{cl} /$ rehuehome/ facultad/ publicaciones/ semiotica/ semiotica2/ index. html. Ed. Facultad de Ciencias Sociales, Universidad de Chile. 
JARA, RENÉ (1972). «La Escuela de Praga y la Teoría Literaria». Problemas de

Literatura 2. Valparaíso: Ed. Universitarias de Valparaíso.

JoFré, MANuEl (1984). «Lectura de Residencia en la tierra, de Pablo Neruda». En «Actas III Seminario Nacional de Estudios Literarios. Temuco: Ed. Universidad de la Frontera.

- (1985). «Semiótica de la lectura». Trilogía 5.94. Santiago: Ed. Instituto Profesional de Santiago.

- (1987). «Poética de la literatura post-estructuralista». En Literatura y Lingüística. Santiago: Universidad Católica Blas Cañas.

- (1987). «El dilema de la teoría literaria actual». Estudios Filológicos 22. Valdivia: Ed. Universidad Austral.

- (1995). Tentando vías: semiótica, estudios culturales y teoría de la literatura. Santiago: Ediciones Universidad Católica Blas CañasUniversidad Andina Simón Bolivar.

LUCO, NANCY (1996). Descripción del funcionamiento de una organización: reconstrucción de la utopíal realidad. Santiago: Tesis Magistratura en Comunicación, Universidad de Chile

MATTElarT, ARMAND (1969). «Prefiguración de la ideología burguesa». Cuadernos de la Realidad Nacional 1. Santiago: Ed. Universidad Católica.

- (1970a). «El marco del análisis ideológico». Cuadernos de la Realidad Nacional 3. Santiago: Ed. Universidad Católica.

- (1970b). «Estructura del poder informativo y dependencia». Cuadernos de la Realidad Nacional 3. Santiago: Ed. Universidad Católica.

- (1970c). «La mitología de la juventud en un diario liberal». Cuadernos de la Realidad Nacional 3. Santiago: Ed. Universidad Católica.

- (1971). «¿Hacia una cultura de la movilización cotidiana? Cuadernos de la Realidad Nacional 10. Santiago: Ed. Univ. Católica.

MATtelart, Michèle (1970a). «El nivel mítico de la prensa seudo-amorosa».

Cuadernos de la Realidad Nacional 3. Santiago: Ed. Univ. Católica.

- (1970b). «El conformismo revoltoso de la canción popular». Cuadernos de la Realidad Nacional 5. Santiago: Ed. Univ. Católica.

MElÉnDEZ, Claudio (1985). «Efectos de la programación con LOGO en el desarrollo de la cognición de niños de 10, 11 y 12 años». Generación 2000 2.2. La Serena: Ediciones Universidad de La Serena.

- (1996a). «Algunas implicaciones ideológicas del proceso de enseñanza de lenguas extranjeras». En Actas del Décimo Encuentro Nacional de SONAPLES. Iquique: Ediciones Universidad Arturo Prat.

- (1996b). «Caracterización del rol de la semántica dentro de la linguiística». Revista Chilena de Semiótica 2. Santiago, Internet: http: // rehue. csociales. uchile. $\mathrm{cl} /$ rehuehome/ facultad/ publicaciones/ semiotica/ semiotica2/ index. html. Ed. Facultad de Ciencias Sociales, Universidad de Chile.

MONTECINO, SONIA (1988). Organizaciones lideres y contiendas mapuches», en colaboración con Rolf Foester. Santiago: Ediciones CEM. 
- (1992). Espejos y travesía. Mujer y antropología en los 90, co-editora con Regina Rodríguez. Santiago: Ediciones ISIS Internacional.

- (1993). Sangres cruzadas, mujeres chilenas y mestizaje. Santiago: Ediciones SERNAM.

- (1994). Ritos de vida y muerte. Brujas y hechiceras. Santiago: Ediciones SERNAM.

- (1996a). Sol viejo, sol vieja. Lo femenino en la cultura mapuche. Santiago: Ediciones SERNAM.

- (1996b). Modelando el barro. Celos y sueños de la alferería. Santiago: Ediciones SERNAM.

- (1996c). Madres y huachos. Alegorías del mestizaje chileno. Buenos Aires: Editorial Sudamericana.

Montesinos, Elisa; Ponce, Marcela; Panger, Ibi; Delgado, Anamaría (1996). «Análisis de textos esquizofrénicos». Revista Chilena de Semiótica 2. Santiago, Internet: http: // rehue. csociales. uchile. cl/ rehuehome/ facultad/ publicaciones/ semiotica/ semiotica2/ index. html. Ed. Facultad de Ciencias Sociales, Universidad de Chile.

Mora, Georgina (1996). Descripción de un telediario regional. Santiago: Tesis de Magistratura en Comunicación Social, Universidad de Chile.

Morel, C.; Ossandon, F., y Fuenzalida, V. (1972). «Más allá de la entretención de las teleseries. Búsqueda de la ideología en Bonanza y F.B.I. en Acción». Revista EAC. Santiago: Escuela de Artes de la Comunicación, Universidad Católica de Chile.

MÜHLHAUSER, GRETEL (1996). «El imaginario de lo masculino y femenino en estudiantes de pedagogía». Revista Chilena de Semiótica 1. Santiago, Internet: http: // rehue. csociales. uchile. cl/ rehuehome/ facultad/ publicaciones/ semiotica/ semiotica2/ index. html. Ed. Facultad de Ciencias Sociales, Universidad de Chile.

Munizaga, Gisélle; RIVERA, ANNY (1983). La investigación en comunicación social en Chile. Lima: Ed. Desco/Ceneca.

MunIZAGA, Giséle (1972a). «Algunas ideas sobre lo ideológico en el cine». Revista EAC 2. Santiago: Escuela de Artes de la Comunicación, Universidad Católica de Chile.

- (1972b). «¿Cuántas patas tiene un gato?». Revista EAC. Santiago: Escuela de Artes de la Comunicación Universidad Católica.

- (1975). «La teleserie policial: una moral de la violencia». Cuadernos de Estudio 6. Santiago: Escuela de Artes de la Comunicación, Universidad Católica.

MuÑoz, LuIS (1975). «La noria, poema de Antonio Machado». Acta Literaria

1. Concepcion.

- (1975). «El drama: una descripción formal». Estudios Filológicos 11. Valdivia: Ediciones Universidad Austral.

- (1978). «El ensayo como discurso literario». Acta Literaria 3. Concepción: Ediciones Universidad de Concepción. 
OelKer, Dieter (1977). «Análisis de un poema de García Lorca». Acta Literaria 2. Concepción: Ediciones Universidad de Concepción.

- (1978). «Morfología de un relato de Alejo Carpentier». Revista Chilena de Literatura 12. Santiago.

- (1978). «Análisis estructural de un relato de las 1001 noches». Acta Literaria. Concepción: Ediciones Universidad de Concepción.

Pereira, Sergio (1983a). «El texto como máscara». En Actas II Seminario Nacional de Estudios Literarios. Santiago: Universidad de Santiago.

- (1983b). «Ideas para un estudio de la anfibolia en el arte nuevo de hacer comedias en este tiempo». En Actas II Seminario Nacional de Estudios Literarios.

- (1984). «El proceso de secuencialidad en el conflicto dramático de Hamlet». En Actas III Seminario Nacional de Estudios Literarios. Temuco: Ed. Universidad de la Frontera.

PICCINI, MABEL (1970). «El cerco de las revistas de ídolos». Cuadernos de la Realidad Nacional 3. Santiago, Ed. Universidad Católica.

RABANALES, AMBrosio (1976). «Los contenidos fonológicos y su conceptualización en el «Esbozo de una Nueva gramática de la Lengua española». Estudios Filológicos 11. Valdivia: Ed. Universidad Austral.

- (1977). «La categoría gramatical de persona». Estudios Filológicos 12. Valdivia: Ediciones Universidad Austral.

- (1980). «Relaciones asociativas en torno al «Canto Negro» de Nicolás Guillén». Estudios Filológicos 15. Valdivia: Ediciones Universidad Austral.

RIBEIRO, LuIS FELIPE (1970). Apuntes sobre el problema lingüístico en la alfabetización. Santiago: Ed. Secretariado de Comunicación Social (SEDECOS), mimeografiado.

- (1972a). «Sobre la semantización de la sexualidad». Cuadernos de la Realidad Nacional 12. Santiago, Ed. Universidad Católica.

- (1972b). La estructura mítica de los discursos sobre la legalidad. Santiago: Ediciones Universidad Católica, Mimeo.

Rodríguez, Marta (1983). «La modalidad de crítica en Octavio Paz». En Actas II Seminario Nacional de Estudios Literario. Santiago: Ediciones Universidad de Santiago.

SÁEZ, LEOPOLdo (1987). «Los inventarios léxicos automatizados y el español: proposiciones terminologicas». Literatura y Lingüística 1. Santiago: Ed. Universidad Católica Blas Cañas.

SÁNCHez, MAlva (1996). Análisis de las variaciones experimentadas por las publicaciones del servicio agrícola y ganadero (SAG) en el período histórico 1967-1990. Santiago: Tesis de Magistratura en Comunicación Social, Universidad de Chile.

SASSO, ILSE (1979). Europa, Felipe II y El Escorial (relación literario-arquitectónico de contenido). Santiago: mimeo textos de Investigación, Universidad Técnica del Estado. 
- (1980). «Poema Sin Luz, de Vicente Aleixandre». En Encuentro. Santiago, Ed. Universidad de Santiago.

SCHUlTZ, MARGARITA (1989). El significado en la música. Santiago: Dolmen Ediciones.

- (1992). Epistemología del arte. Santiago: Ediciones Facultad de Artes, Universidad de Chile.

- (1996). «La notación musical desde la perspectiva peirciana». Revista Chilena de Semiótica 1. Santiago, Internet: http: // rehue. csociales. uchile. cl/ rehuehome/ facultad/ publicaciones/ semiotica/ semiotica2/ index. html. Ed. Facultad de Ciencias Sociales, Universidad de Chile.

Sillva, María InÉs (1971). Semiología del western. Valparaíso: Ediciones Previas ICSD/UCV.

TriviÑos, GLbERTo (1975). «La destrucción del verosímil folletinesco en Boquitas pintadas, de Manuel Puig. Acta literaria 1. Concepción: Ediciones Universidad de Concepción.

VALlEJOS, GUIDO (1985). «La semiótica de la significación o el tránsito desde el código hacia el hipercódigo». Trilogía 5.9. Santiago: Ed. Instituto Profesional de Santiago.

- (1987). «Algunas bases filosóficas de la pragmática lingüística». Lenguas Modernas 14. Santiago: Ed. Universidad de Chile.

- (1991). «La teoría representacional de la mente y el rol causal del contenido intencional». Lenguas Modernas 18. Santiago, Ed. Universidad de Chile.

VARELA, José (1972). «La linguíística y los problemas de la traducción». Problemas de Literatura 2. Valparaíso: Ed. Universitarias de Valparaíso.

WAGNER, Claudio (1977). «Estado actual de los estudios semánticos europeos». Estudios Filológicos 12. Valdivia: Ed. Universidad Austral.

- (1981). «El mecanismo semántico de la metáfora». Estudios Filológicos 16. Valdivia.

- (1988). «Programa de investigación sobre la enseñanza de la lengua materna en Chile». Estudios Filológicos 23. Valdivia: Ediciones Universidad Austral.

Walther, Elisabeth (1994). Teoría de los signos. Traducción de Margarita Schultz y Jaime Cordero. Santiago: Dolmen Ediciones.

Waisman, LuIs; Donoso, Nelly; Thomas, Eduardo (1978). Método para análisis e interpretación de la obra dramática. Santiago: mimeo, Universidad de Chile.

Waisman, Luis (1974). Semiología Arquitéctonica. Santiago: Ed. Fac. de Arquitectura y Urbanismo, Universidad de Chile.

- (1983). «Sobre el concepto de espectáculo». En Actas II Seminario Nacional de Estudios Literarios. Santiago: Ed. Universidad de Santiago.

- (1984). «Sobre las ideas estéticas de José Ortega y Gasset». Estudios Filológicos 19. Valdivia: Ed. Universidad Austral. 